\title{
Non-equilibrium quasi-stationary states in a magnetized plasma
}

\author{
K. Rypdal, J.-V. Paulsen, O. E. Garcia, S. V. Ratynskaia, and V. I. Demidov \\ Department of Physics, University of Troms $\emptyset, 9037$ Troms $\emptyset$, Norway \\ Received: 4 January 2002 - Revised: 23 September 2002 - Accepted: 4 October 2002
}

\begin{abstract}
Non-equilibrium quasi-stationary states resulting from curvature driven interchange instabilities and driftwave instabilities in a low beta, weakly ionized, magnetized plasma are investigated in the context of laboratory experiments in a toroidal configuration. Analytic modelling, numerical simulations and experimental results are discussed with emphasis on identifying the unstable modes and understanding the physics of anomalous particle and energy fluxes and their linkage to self-organized pressure profiles.
\end{abstract}

\section{Introduction}

Since K. Birkeland's pioneering terrella experiments during the first two decades of the $20^{\text {th }}$ century (Rypdal and Brundtland, 1998) laboratory experiments have been carried out to study processes that are assumed to be essential for the global dynamics of the solar-terrestrial system. Birkeland's experiments attempted to model the global system itself, but it has later been shown that it is impossible to scale all relevant physical parameters down to laboratory dimensions (Block, 1967). Many laboratory physicists believe that the scaling problem might invalidate laboratory simulations of global space systems, but insist that there is still a strong case for laboratory investigation of micro-processes. Experiments of this type should be carefully designed to admit quiescent equilibrium states, which then can be tuned to onset of instability and turbulence. In this way the linear physics can be fully tested, and linear and non-linear evolution of instabilities can be studied and compared to theoretical predictions. Examples of experimental devices that have served this purpose are Q-machines (Motley, 1975), where the resistive drift waves were first discovered, and certain large linear devices (Gekelman, 1999), which have facilitated fundamental studies of Alfvénic phenomena.

Correspondence to: K. Rypdal

(kristoffer.rypdal@phys.uit.no)
One should bear in mind, however, that this experimental focus on the linear dynamics and the transition from stable to unstable equilibria does not let us study all aspects of the complex dynamics that typically govern space systems, nor is it suitable for studying all relevant instabilities. One example is a class of low-frequency instabilities in magnetized plasma called interchange instabilities. Such instabilities excite field-aligned structures (flute modes) and are driven by a pressure gradient opposing either either a gravity force or a centrifugal force due to magnetic field curvature. They are important in ionospheric dynamics, manifested for instance in the so-called equatorial spread $\mathrm{F}$ irregularities, which take their name from the spreading of ionograms obtained from equatorial locations (Hysell, 2000). Spread F is now known to be caused by interchange instabilities driven by the steep density gradient in the night-time equatorial Fregion. Although only the bottomside F-region is linearly unstable, the nonlinear stage of the instability leads to penetration of plumes through the F peak and well into the top side. In this manner the instabilities lead to strong transport and mixing which is of fundamentally nonlinear nature.

Gravity is unimportant in laboratory plasmas, so the study of these phenomena requires a curved magnetic field, while the quiescent laboratory plasmas require a straight field. The problem is that the bending of a simple straight magnetic field not only creates a loss of stability, but also a loss of MHD-equilibrium. In toroidal magnetic confinement devices equilibrium is restored by the introduction of a magnetic rotational transform, but this modification also suppresses the interchange instabilities. Thus, neither the quiescent linear devices, nor the toroidal confinement devices are suitable for studying such phenomena.

The philosophy underlying the study of quiescent configurations for gaining understanding of space systems is based on an implicit assumption that the time-averaged state necessarily corresponds to an MHD-equilibrium. However, natural plasmas are often found in quasi-stationary states for which the time-averaged state is not an equilibrium. Such plasmas develop strong stationary or fluctuating flows, and 
force balance is maintained only by inclusion of inertial and/or friction forces. The simplest experimental configuration for studying such plasma states is the simple magnetized torus (Rypdal et. al., 1994), a configuration where a plasma is immersed in an externally generated toroidal magnetic field without the rotational transform that is necessary for MHD equilibrium and stability.

At this point it may be necessary to make some comments on the notions of equilibrium and stability. Strictly speaking instabilities can only develop on the background of an equilibrium situation, and the notion of instability has no meaning if an equilibrium does not exist. Nevertheless, plasma instabilities are often identified in a system with fluctuations, even when the equilibrium state from which the unstable perturbations grow cannot be uniquely defined. What is identified is the instability mechanism and the fluctuation modes, which may persist also in the nonlinearly saturated state. It will be shown in this paper that a stationary flow can result from the same mechanism that drives the growth of a particular unstable perturbation of an equilibrium state. It is a matter of definition whether such a flow should be conceived as a particular saturated state of the instability or a stationary and stable equilibrium flow. We prefer the former definition, because the stationary flow states and the quasistationary turbulent states are all governed by the same physical mechanisms. We call a state quasi-stationary if the fluctuating density, temperature and fluid velocity are considerably smaller than their time-averaged values, and if the fluctuations are stationary in the sense that the ensemble mean and auto-correlation are independent of time. In this case it is meaningful to think of the time-averages of these quantities as background profiles.

In a non-equilibrium state the plasma production mechanism and the boundary conditions in general force the plasma system to accomodate fluxes of matter, energy and entropy which cannot be maintained in a quiescent state. The classical turbulent transport paradigm could be described as follows: Imposed fluxes universally give rise to gradients in configuration- and/or velocity-space, or to stretching and twisting of magnetic field lines. Such gradients or distortions provide the free energy that feed plasma instabilities, and the resulting fluctuations give rise to the transport necessary to maintain the imposed fluxes. The linear growth rate of the instability normally increases monotonically with increasing gradient, and often there is an instability threshold in the sense that instability occurs only when the gradient exceeds a certain value. For a given imposed flux the gradient is given by the negative feedback loop; increased gradient $\rightarrow$ increased growth rate $\rightarrow$ higher fluctuation levels $\rightarrow$ increased anomalous transport $\rightarrow$ reduced gradient. If there is no threshold, the relaxed gradient depends strongly on the magnitude of the imposed flux. In the presence of an instability threshold, however, the gradients will usually remain close to this threshold. This concept of a critical gradient is very useful, although somewhat over-simplified, since the stability of the system may depend on the global profile of plasma parameters, not only on local gradients. In this picture the growth of the unstable modes could in principle saturate before they reach nonlinear levels because of the flattening of the profiles, in analogy with the plateau formation of the particle velocity in quasilinear theory of velocityspace instabilities. The quasilinear picture of turbulence implies the existence of a characteristic spatial scale (i.e. the wavelength of the most unstable mode), which appears in the anomalous transport coefficient, and the dynamics of the quasilinear transport is a diffusion process. The quasilinear transport paradigm has been challenged by observations from a variety of nonequilibrium quasistationary plasmas indicating absence of characteristic scales of the fluctuations. The scale invariance is often manifested in the power-law behaviour of Fourier energy density spectra of the turbulence. Power-law spectra $S(f) \sim f^{-\gamma}$ at low frequencies have been observed in signals from magnetic confinement plasmas and space plasmas like those in planetary magnetotails and various astrophysical objects. Such spectra are associated with an algebraic tail in the autocorrelation function $C(\tau) \sim \tau^{\gamma-1}$. If $\gamma>0$, the integral $\int_{0}^{\infty} C(\tau) d \tau$ diverges, and the fluctuations are said to exhibit long-range correlations.

The paper is organized as follows. In Sect. 2 we describe the main cross field transport mechanisms for plasma and charge in a low beta and weakly ionized plasma with curved magnetic field, and the physics that determines the plasma potential profile in a hot cathode toroidal discharge is outlined. Section 3 contains a systematic derivation of a reduced fluid model which contains all the essential physics describing the source driven non-equilibrium quasi-stationary state of such a plasma. A discussion of the (lack of) equilibrium for the simple toroidal plasma configuration and the implications for the resulting plasma state is given in Sect. 4. Section 5 starts with a summary of known results of local linear stability analysis for flute interchange modes and drift waves, followed by some results of global two-dimensional (2-D) numerical simulations of the model derived in Sect. 3 in the flute mode limit. Stationary and quasi-stationary (turbulent) flow states are identified and the existence of critical (resilient) pressure profiles are demonstrated numerically as well as experimentally. In Sect. 5 we also present flute mode simulations in a slab geometry. Here critical profiles are demonstrated very clearly, and shown to correspond to a linear stability threshold. The plasma potential and electron density fluctuations are shown to be approximately in anti-phase, which is unfavourable for plasma transport. Section 6 briefly describe some experimental results on fluctuations and transport which have been discussed in more detailed in a recent publication (Ratynskaia et al., 2002). These results are contrasted with the simulations of Sect. 5, and the role of flute modes and drift waves are discussed.

\section{Physics of the simple torus}

A valuable supplement to the study of anomalous transport in fusion magnetic confinement devices are experiments on 
low frequency instabilities and turbulent transport conducted in cold, magnetized plasma where electrostatic probes can be operated as the main diagnostic. As mentioned in Sect. 1 a configuration that allows excitation of interchange instabilities is the simple torus configuration (Rypdal et. al., 1994). This device is simpler than fusion devices in most respects. The magnetic geometry is purely toroidal, simplifying the modelling considerably. Plasma beta is very low, justifying an elecrostatic description of all phenomena. The ions are very cold, allowing us to neglect ion pressure. The electrons are also quite cold, and not very dense, so probes can be used as a diagnostic also in the core plasma. And the device is operated in a steady state mode, so device operation represents no limit to the amount of data that can be aqcuired from the experiment.

The plasma in this geometry is most conveniently described in cylindrical cordinates $(R, \varphi, z)$, where $z$ is the coordinate along the major torus axis, $R$ is the distance from this axis, and $\varphi$ is the azimuthal (toroidal) angle. By convention we chose the azimuthal unit vector to be in the opposite direction of the magnetic field, i.e. $\mathbf{B}=-\left(B_{0} R_{0} / R\right) \hat{\boldsymbol{\varphi}}$.

\subsection{Basic concepts of anomalous transport}

In a low beta plasma the time-averaged cross-field anomalous particle flux density $\Gamma_{\perp}=\left\langle n \mathbf{v}_{\perp}\right\rangle$ is due to electrostatic oscillations in plasma density $n$ and electric field $\mathbf{E}=-\nabla_{\perp} \phi$. The latter gives rise to fluctuations in the plasma drift velocity $\mathbf{v}_{\perp} \approx \mathbf{v}_{E}=\nabla_{\perp} \phi \times \hat{\varphi} / B$ which follows from Ohm's law $\nabla_{\perp} \phi=\mathbf{v} \times \mathbf{B}$ for a perfectly conducting medium. The plasma beta $\beta=\mu_{0} p / B^{2}$ is of order $10^{-5}$ in these experiments, so the total magnetic field can accurately be represented by the field $\mathbf{B}=-\left(B_{0} R_{0} / R\right) \hat{\boldsymbol{\varphi}}$ generated by the external field coils. In Sect. 5 it is shown that in this configuration the electrostatic interchange instability generates either a stationary flow in the form of a double vortex which transports plasma from the center to the wall by passive advection, or a fluctuating state dominated by poloidally rotating, electrostatic field-aligned vortices. In the fluctuating state the vortex dynamics is responsible for a major part the radial mass transport as well as important contributions to the cross-field current. At an arbitrary location in the toroidal plasma column let us consider the flux component along the minor radius, $\Gamma_{x}=\left\langle n v_{x}\right\rangle$, where $v_{x}=E_{y} / B$ is the $\mathbf{E} \times \mathbf{B}$ velocity in this direction. Here $E_{y}=-\partial_{y} \phi$ is the electric field component in the poloidal direction. By Fourier expanding $n$ and $\phi$ in the $y$-coordinate, we find for the flux density averaged over the poloidal coordinate $\Gamma_{x 0}=-(2 / B) \sum_{k>0} k\left\langle\left|n_{k} \phi_{k}^{*}\right|\right\rangle \sin \alpha_{k}^{(n \phi)}$, where $\alpha_{k}^{(n \phi)}=$ $\arg \left\langle n_{k} \phi_{k}^{*}\right\rangle$ is the cross-phase between $n_{k}$ and $\phi_{k}$. Essential for the transport is the average phase difference between $n_{k}$ and $v_{k}=i k \phi_{k} / B$ for the Fourier components of the fields. One observes that the turbulent flux is proportional to the sine of the cross-phase, and hence it vanishes if $n_{k}$ and $\phi_{k}$ are exactly in phase, or exactly in antiphase. Cross-field currents can be diamagnetic, inertial, or collisional, as can be shown by writing the one-fluid momentum equation in the form

$\mathbf{j} \times \mathbf{B}=\nabla p+\rho \frac{d \mathbf{v}}{d t}+\rho v_{i n} \mathbf{v}$.

Equation (1) shows that the sources of a cross-field current density $\mathbf{j}$ are forces due to gradients in the pressure $p$, inertial forces due to accelerations, and friction forces due to an ionneutral collision frequency $v_{i n}$. Thus, by taking the crossproduct of Eq. (1) by $\mathbf{B}$, the perpendicular current density can be written as $\mathbf{j}_{\perp}=\mathbf{j}_{p}+\mathbf{j}_{m}+\mathbf{j}_{v}$, where $\mathbf{j}_{p}=B^{-1} \nabla p \times$ $\hat{\boldsymbol{\varphi}}, \mathbf{j}_{m}=B^{-1} \rho d_{t} \mathbf{v} \times \hat{\boldsymbol{\varphi}}$, and $\mathbf{j}_{v}=B^{-1} v_{i n} \rho \mathbf{v} \times \hat{\boldsymbol{\varphi}}$.

It will be shown in Sect. 3.1 that for a wide range of plasma parameters we can invoke the quasineutral approximation, which imples that $\nabla \cdot \mathbf{j}=0$ even for the instantaneous current density. Thus, an approximate evolution equation is

$$
\nabla \cdot\left(\mathbf{j}_{m}+\mathbf{j}_{p}+\mathbf{j}_{v}\right)=-\nabla \cdot \mathbf{j}_{\|} .
$$

If $\mathbf{v}$ is approximated by the $\mathbf{E} \times \mathbf{B}$ velocity $\mathbf{v}_{E}$, Eq. (2) is an evolution equation for the plasma potential $\phi(\mathbf{x}, t)$. For axisymmetric perturbations (flute mode turbulence) the term on the right hand side of Eq. (2) vanishes, while for for drift modes it depends on the parallel electron conductivity and the parallel wave number $k_{\|}$. In Sect. 3.4 we present a closed model suitable for numerical simulation, consisting of this evolution equation coupled with the mass continuity equation and an equation of state.

If the plasma is in a state of stationary turbulence, the timeaveraged current $\left\langle\mathbf{j}_{p}\right\rangle$ is found by the substitution $p \rightarrow\langle p\rangle$ in the definition of $\mathbf{j}_{p},\left\langle\mathbf{j}_{m}\right\rangle$ by the substitution $\rho d_{t} \mathbf{v} \rightarrow \nabla$. $\langle\rho \mathbf{v v}\rangle$, and $\left\langle\mathbf{j}_{v}\right\rangle$ by the substitution $\rho \mathbf{v} \rightarrow\langle\rho \mathbf{v}\rangle$. The averaged inertia current $\left\langle\mathbf{j}_{m}\right\rangle$ can be considered to be driven by the dynamic stress dyad $\mathbf{T}_{m}=\langle\rho \mathbf{v v}\rangle$. In the drift-approximation, where $\mathbf{v}$ in $\mathbf{T}_{m}$ is approximated by $\mathbf{v}_{E}$, the inertial current can be recognized as the current arising from the ion polarization drift. The dynamic stress tensor has a contribution from the average flow $\mathbf{T}_{m 0}=\langle\rho\rangle\langle\mathbf{v}\rangle\langle\mathbf{v}\rangle$ and a contribution from the fluctuations in the flow, the latter is known as the Reynolds stress $\mathbf{T}_{R}=\mathbf{T}_{m}-\mathbf{T}_{m 0}$. In a poloidally rotating plasma the resulting stress (the centrifugal force) gives rise to a poloidal inertia current, and only the Reynolds stress can give rise to a radial component. The current $\mathbf{j}_{v}$ driven by the ion-neutral collisions can also be modified by the drift-approximation $\mathbf{v} \approx \mathbf{v}_{E}$ to yield $\mathbf{j}_{v} \approx-\sigma_{P} \nabla_{\perp} \phi$, where $\sigma_{p}=\rho v_{i n} / B^{2}$ is known as the Pedersen conductivity. In a two-fluid model this current arises from the perpendicular ion mobility due to ion-neutral collisions.

From Eq. (2) we can draw some important conclusions about the nature of the time-averaged plasma state. In the presence of a stationary, turbulent flow, the charge continuity equation yields $\nabla \cdot\left\langle\mathbf{j}_{\perp}+\mathbf{j}_{\|}\right\rangle=0$. If the time-averaged state is axisymmetric, we have that $\nabla \cdot\left\langle\mathbf{j}_{\|}\right\rangle=\partial_{\varphi}\left\langle j_{\|}\right\rangle=0$, hence the time-average of Eq. (2) takes the form

$$
\frac{2}{B R} \frac{\partial\langle p\rangle}{\partial z}=\nabla \cdot\left(\frac{\mathbf{B} \times \nabla \cdot \mathbf{T}_{m}}{B^{2}}\right)-v_{i n} \nabla \cdot\left(\frac{\left\langle\rho \nabla_{\perp} \phi\right\rangle}{B^{2}}\right) .
$$

For a static situation $(\mathbf{v}=0)$ both terms on the right hand side in Eq. (3) vanish, so equilibrium is possible only if 
$\partial_{z}\langle p\rangle=0$. This means that a magnetostatic equilibrium with $p=0$ on the boundaries cannot exist. An equilibrium in the sense of a stationary, or stationary turbulent, flow can only be provided by the currents $\left\langle\mathbf{j}_{m}\right\rangle$ and $\left\langle\mathbf{j}_{v}\right\rangle$ due to inertial stress and/or the collisional friction. The charge accumulation from these currents is represented by the two terms on the right hand side of Eq. (3). It will be shown in Sect. 4 that global charge- or force-balance can only be obtained if plasma currents are allowed to close along the vessel walls or a poloidal limiter.

\subsection{Potential profile and poloidal rotation}

The insights presented here have evolved from experiments on plasma discharges in the simple torus "Blaamann" (Rypdal et. al., 1994). A standard method of plasma production in a simple torus is by electron emission from a hot negatively biased cathode located near the center line (minor axis) of the toroidal plasma column. This implies that negative charge is injected in a toroidal magnetic flux tube far from the bounding walls. The charge accumulating in this flux tube can only be compensated by a cross-field electric current flowing inwards from the wall. Rypdal et al. (1996) have shown that the contribution to the total current from the pressure force and the inertial force from the stationary flow vanishes, hence the discharge current must be anomalous for low neutral gas pressures $\left(<10^{-2} \mathrm{~Pa}\right)$ (Rypdal et al., 1997), but may be due to ion-neutral collisions for high pressures.

In the experiments a negative potential well is formed with nearly circular equipotentials in a poloidal cross-section, and the potential minimum is located where the flux tube through the cathode intersects this poloidal cross-section plane. The potential well gives rise to a poloidal plasma rotation with a strongly sheared flow in certain regions. This suggests that a radial electric field, and hence a poloidal flow $\mathbf{v}_{E}=\nabla \phi \times$ $\hat{\boldsymbol{\varphi}} / B$ is neccessary to maintain the cross-field current. In the straight cylinder limit $(R \rightarrow \infty)$, and a cylinder-symmetric, laminar poloidal flow, $v_{\theta}=\partial_{r} \phi / B, \mathbf{j}_{p}$ and $\mathbf{j}_{m}$ are poloidal and $\mathbf{j}_{v}$ is radial. Let us represent the charge injected from the cathode as a source term in the charge continuity equation, $\nabla \cdot \mathbf{j}_{v}=S(r)$, so that $I(r)=-2 \pi \int_{0}^{r} S\left(r^{\prime}\right) r^{\prime} d r^{\prime}>0$ is the current directed towards the cylinder axis per unit length of the cylinder. The expression for $\mathbf{j}_{v}$ can then be integrated to yield

$\phi(r)=-\frac{B^{2}}{2 \pi v_{i n}} \int_{r}^{a} \frac{I d r^{\prime}}{\rho\left(r^{\prime}\right) r^{\prime}}$,

where we have assumed $\phi(a)=0$ at the wall $r=a$. If we assume that $S$ is localized to the cylinder axis, i.e. if $S(r)=(2 \pi r)^{-1} \delta(r)$, then $I(r)=I_{0}$ for all $r>0$, where $I_{0}$ is the discharge current per unit length of the cylinder. The potential profile calculated from Eq. (4) agrees very well with the one measured experimentally when electron density measurements (needed to estimate $\left.\rho\left(r^{\prime}\right)\right)$ are made by cylindrical Langmuir probes employing kinetic theory for the electron saturation current in a magnetized plasma (Demidov et al., 1999). This actually serves as a validation of this method of electron density measurement, which yields values more than 3 times higher than results based on the conventional Langmuir formula for unmagnetized plasmas.

In the real experiment the charge source is also usually a plasma source, since the injected electron also gives rise to ionization. The average number of ionizations per injected electron depends on the discharge voltage. Numerical simulations described in Sect. 5.2 confirm the relaxed potential profiles described by Eq. (4), and they also show that the onaxis plasma density grows linearly due to inefficient radial plasma transport, until the radial density scale-length exceeds some kind of threshold. At this threshold the plasma goes turbulent, and the averaged density gradient remains close to this threshold during the subsequent evolution.

\section{Fluid models}

The global properties of toroidal plasmas are usually studied within the framework of the MHD-model, while the phenomena associated with plasma transport frequently is studied within the electrostatic approximation. In very low beta plasmas (in the typical simple torus experiments $\beta \sim 10^{-5}$ ) the electrostatic approximation is always valid. The phenomena we deal with in this paper, plasma equilibrium, flute modes and drift waves, can all be described within the framework of a one-fluid model, although the standard approach is to use a two-fluid model. The former has some appeal, since a formulation in terms of a one-fluid flow and a charge flow (current density) links the electrostatic field directly to the charge continuity equation, and the contributions from ionization and charge injection due to electrons emitted from a hot cathode are naturally represented as source terms in these equations.

\subsection{The full one-fluid model}

By addition of the momentum equations for electrons and ions in a weakly ionized plasma we find a slightly generalized version of Eq. (1),

$\rho \frac{d \mathbf{v}}{d t}=-\nabla p+\mathbf{j} \times \mathbf{B}+\varrho \mathbf{E}-\rho \nu \mathbf{v}-\rho \frac{m_{e}}{m_{i}} v_{e n} \frac{\nabla p_{e} \times \mathbf{B}}{e n B^{2}}$. (5)

Here $\mathbf{v}=\left(m_{i} \mathbf{v}_{i}+m_{e} \mathbf{v}_{\mathbf{e}}\right) /\left(m_{i}+m_{e}\right)$ is the one-fluid velocity, where $\mathbf{v}_{e}$ and $\mathbf{v}_{i}$ are the electron and ion fluid velocities, and $m_{e}$ and $m_{i}$ the corresponding masses. The quantity $p=p_{e}+p_{i}=n T_{e}+n T_{i}$ is the total scalar pressure and $\varrho$ is the charge density. The effect of collisions between electron and ions with neutrals are expressed through the collision frequencies $v_{e n}$ and $v_{i n}$, and we have introduced the effective collision frequency $v=v_{i n}\left(1+\epsilon_{n}\right)$, where $\epsilon_{n}=m_{e} v_{e n} / m_{i} v_{i n}$ is the ratio between electron and ion mobilities which is of order $10^{-1}$ for a Helium plasma, and of order $10^{-2}$ for Argon. In standard one-fluid formulation the remaining information in the momentum equations is expressed in Ohm's law. For our purpose we express this most conveniently by employing the ion momentum equation to 
obtain Ohm's law perpendicular to the magnetic field. By assuming $\mathbf{v}_{e \perp} \sim \mathbf{v}_{i \perp}$, implying $\mathbf{v}_{i \perp}=\mathbf{v}_{\perp}\left[1+\mathcal{O}\left(m_{e} / m_{i}\right)\right]$, the perpendicular Ohm's law takes the form

$\mathbf{E}_{\perp}+\mathbf{v}_{\perp} \times \mathbf{B}=\eta_{\perp} \mathbf{j}+\frac{m_{i}}{e}\left(\frac{d}{d t}+v_{i n}\right) \mathbf{v}_{\perp}+\frac{\nabla p_{i}}{e n}$,

and assuming that $v_{e \|} \gg v_{i \|}$, implying $v_{e \|} \approx-j_{\|} / e n$, we get from the electron momentum equation the parallel Ohm's law;

$E_{\|}=\eta j_{\|}-\frac{\nabla_{\|} p_{e}}{e n}$.

Here $\eta_{\perp}=\left(m_{e} / e^{2} n\right) v_{e i \perp}$ is the perpendicular Spitzer resistivity, and $\eta=\left(m_{e} / e^{2} n\right)\left(v_{e i}+v_{e n}\right)$. The remaining fluid equations are the mass continuity equation and the energy equation,

$$
\begin{aligned}
& \frac{\partial \rho}{\partial t}+\nabla \cdot(\rho \mathbf{v})=0, \\
& \frac{d}{d t}\left(p \rho^{-\gamma}\right)=0 .
\end{aligned}
$$

We have chosen to represent the energy equation by the adiabatic equation of state (9), but other closures are of course possible.

We shall invoke the quasi-neutral approximation, which amounts to neglecting the electric force on space charge $\varrho \mathbf{E}$ in Eq. (5) and the displacement current in Ampère-Maxwell's law, the latter being equivalent to neglecting $\partial \varrho / \partial t$ in the equation $\partial \varrho / \partial t+\nabla \cdot \mathbf{j}=0$. In Goldston and Rutherford (1995) it is shown that this approximation is valid if $\chi^{-1} \equiv \epsilon_{0} B^{2} / \rho=\lambda_{D}^{2} / \rho_{s}^{2} \ll 1$, where $\chi$ is the effective electric susceptibility of the plasma, $\lambda_{D}$ is the Debye length, and $\rho_{s}$ is the ion Larmor radius with electron temperature. This condition is valid for most magnetized laboratory and space plasmas.

Another approximation we shall make to simplify the discussion is to assume cold ions, so that the ion pressure $p_{i}$ can be neglected compared to the electron pressure, and we can put $p_{e} \approx p$ in Eqs. (5) and (7). For typical experimental conditions, however, the ion pressure term on the right hand side of Eq. (6) is not negligible compared to the other terms on the right hand side, and it will be treated on the same footing as these terms in the following.

\subsection{The drift expansion}

The drift expansion can be invoked if the terms on the right hand side of Eq. (6) are small compared to each of the terms on the on the left hand side, implying that $\mathbf{v}_{\perp} \approx \mathbf{v}_{E}$. This is true if the temporal and perpendicular spatial scales of the phenomena in question are given by $\omega$ and $k$, and we have that $\omega, k_{\perp} v_{\perp}$, and $v_{i n}$ are all much smaller than the ion cyclotron frequency $\omega_{c i}=e B / m_{i}$. In addition we require that $\nabla_{\perp} p_{i} / e n E_{\perp} \sim T_{i} / e \phi$ and $\eta j_{\perp} / E_{\perp} \sim\left(T_{e} / e \phi\right)\left(v_{e i} / \omega_{c e}\right)$ are both small compared with unity. For the typical simple torus experiment $\omega / \omega_{c i} \sim v_{i n} / \omega_{c i} \sim 10^{-2}$. The ratios $k_{\perp} v_{\perp} / \omega_{c i}$ and $T_{i} / e \phi$ can of course also be determined from the experiments, but there are also some theoretical leads to estimate them. The ion-neutral collisions (charge exhange) ( $v_{i n} \sim 5 \times 10^{4} \mathrm{~s}^{-1}$ ) tend to cool the ions towards the neutral gas temperature. However, after a charge exchange collision the $\mathbf{E} \times \mathbf{B}$ mechanism accelerates ions to the $v_{E}$ velocity within one gyroperiod, and these velocities are randomized due to collisions with charged particles. The result is a drifting Maxwellian ion fluid with thermal velocity $v_{T i} \equiv\left(T_{i} / m_{i}\right)^{1 / 2} \sim v_{E} \sim k_{\perp} \phi / B$. If we define the finite ion Larmor radius parameter as $\delta_{i} \equiv k_{\perp} \rho_{i}$, this implies that $k_{\perp} v_{\perp} / \omega_{c i} \sim T_{i} / e \phi \sim \delta_{i}$. The ratio $\left(T_{e} / e \phi\right)\left(v_{e i} / \omega_{c e}\right)$ is much smaller than $\delta_{i}$ under all conditions of experimental interest, so perpendicular electron resistivity can safely be neglected. In our laboratory experiments we have $\delta_{i} \sim 10^{-2}$ for the dominant wavenumbers, hence the leading terms on the right hand side in Eq. (6) are those of order $\delta_{i}$. When $\delta_{i} \ll 1$ Eq. (6) can be solved by iteration, which yields

$\mathbf{v}_{\perp}=\mathbf{v}_{E}+\mathbf{v}_{\perp}^{(1)}+\mathcal{O}\left(\delta_{i}^{2} v_{T i}\right)$

where $\mathbf{v}_{E}$ is of order $v_{T i}$ and $\mathbf{v}_{\perp}^{(1)}=\mathbf{v}_{m}+\mathbf{v}_{p i}+\mathbf{v}_{v}$ is of order $\delta_{i} v_{T i}$. Here $\mathbf{v}_{m}=\left(B \omega_{c i}\right)^{-1} D_{t} \mathbf{E}_{\perp}$ is the inertia drift (ion polarization drift), $\mathbf{v}_{p i}=\left(e n B^{2}\right)^{-1} \mathbf{B} \times \nabla p_{i}$ is the ion diamagnetic drift, and $\mathbf{v}_{v}=\left(B \omega_{c i}\right)^{-1} v_{i n} \mathbf{E}_{\perp}$ is the collisional ion drift giving rise to the cross-field Pedersen current. Note that we have introduced here the notation $D_{t} \equiv \partial_{t}+\mathbf{v}_{E} \cdot \nabla$.

\subsection{The electrostatic model}

The electrostatic model assumes that the induced electric field is small compared to the electrostatic component, the ratio of these components being of the order $(\omega / k v) \beta$. For typical simple torus parameters $\omega / k v \sim 1$ and $\beta \sim 10^{-5}$, so the electric field is electrostatic. A complete set of equations can the be constructed from Eqs. (5)-(9), and the charge continuity equation by replacing $\mathbf{E}$ by $-\nabla \phi$. By invoking the quasineutral approximation, the term $\varrho \mathbf{E}$ in Eq. (5) is neglected, and the charge continuity equation, which follows from the divergence of Ampere's law, takes the form $\nabla_{\perp} \cdot \mathbf{j}_{\perp}=-\nabla_{\|} j_{\|}$. The parallel current $j_{\|}=\mathbf{j} \cdot \mathbf{B} / B$ gives an important contribution to the charge budget if the modes dominating the low frequency fluctuations are drift waves (these have a finite parallel wavenumber so that $\nabla_{\|} j_{\|} \sim$ $\left.k_{॥} j_{\|}\right)$. Since in these cases heat conduction along the field lines may smear out the temperature perturbations, it could be appropriate to replace Eq. (9) with an isothermal equation of state $p=c_{s}^{2} \rho$, where $c_{s}^{2} \equiv T_{e} / m_{i}$ is the (constant) ionacoustic velocity. The ES model constitutes a complete set of equations for the fields, $\mathbf{v}, \mathbf{j}, \phi, \rho$, and $p$.

\subsection{Drift expansion in the electrostatic model}

Invoking the drift expansion iteration in Eq. (6) gave us Eq. (10) for the fluid velocity. Replacing $\mathbf{v}_{\perp}$ by the lowest order drift $\mathbf{v}_{E}$ in Eq. (5) yields

$$
\mathbf{j}_{\perp}=\mathbf{j}_{p}+\left(\mathbf{j}_{m}+\mathbf{j}_{v}\right)\left[1+\mathcal{O}\left(\delta_{i}\right)\right]+\mathbf{j}_{e}^{(D)},
$$


The inertia driven current $\mathbf{j}_{m}=\left(e / m_{i}\right) \rho \mathbf{v}_{m}$ has been slightly redefined by replacing $\mathbf{v}_{\perp}$ by $\mathbf{v}_{E}$, and similarly the Pedersen current $\mathbf{j}_{v}=\left(1+\epsilon_{n}\right)\left(e / m_{i}\right) \rho \mathbf{v}_{v}$ has been generalized by including the effect of electron mobility, The last term, $\mathbf{j}_{e}^{(D)}=$ $\left(v_{e n} / \omega_{c e} B\right) \nabla p$, represents the current due to electron diffusion from collisions with neutrals, and is of order $\epsilon_{n}$ compared to $j_{v}$. Normally, therefore, this term is of the same order as those of order $\delta_{i}$ which have already been neglected in the iteration scheme. In order to include the effects of magnetic field curvature, let us consider a toroidal field in cylinder coordinates $(R, \varphi, z)$. We assume $\mathbf{B}=-\left(B_{0} R_{0} / R\right) \hat{\boldsymbol{\varphi}}$, and we have $\nabla \cdot \mathbf{v}_{E}=-(2 / R B)(\partial \phi / \partial z)$, where $z$ is the direction along the major torus axis and $R$ is the distance from this axis. Furthermore, by introducing the inverse aspect ratio $\epsilon_{A}=a / R_{0}$, where $a$ is the minor radius and $R_{0}$ the major radius of the torus, we obtain

$\nabla \cdot\left(\rho \mathbf{v}_{\perp}^{(1)}\right)=-\frac{1}{B \omega_{c i}} \nabla_{\perp} \cdot\left[\rho\left(D_{t}+v_{i n}\right) \nabla_{\perp} \phi\right]+\mathcal{O}\left(\epsilon_{A}, \delta_{i}\right)$,

$\nabla \cdot \mathbf{j}_{\perp}=-\frac{2}{R B} \frac{\partial p}{\partial z}-\frac{1}{B^{2}} \nabla_{\perp} \cdot\left[\rho D_{t} \nabla_{\perp} \phi\right]+\frac{S_{n}}{B^{2}}+\mathcal{O}\left(\epsilon_{A}, \delta_{i}\right)$,

where $S_{n}=-\left(1+\epsilon_{n}\right) v_{i n} \nabla_{\perp} \cdot\left(\rho \nabla_{\perp} \phi\right)+\left(v_{e n} B / \omega_{c e}\right) \nabla^{2} p$ represents the charge accumulation from currents driven by collisions with neutrals. From Eq. (7) we have, assuming constant $T_{e}$ along field lines,

$\nabla_{\|} j_{\|}=-\frac{T_{e}}{\eta e} \nabla_{\|}^{2}\left(\frac{e \phi}{T_{e}}-\ln \rho\right)$.

Using Eqs. (13), and (14) in the charge continuity equation, we find

$\nabla \cdot\left(\rho D_{t} \nabla_{\perp} \phi\right)=-\frac{2 B}{R} \frac{\partial p}{\partial z}-\frac{T_{e} B^{2}}{\eta e} \nabla_{\|}^{2}\left(\frac{e \phi}{T_{e}}-\ln \rho\right)+S_{n}$.

Using Eq. (10) the mass continuity equation (8) can be written as $D_{t} \rho=-\rho \nabla \cdot \mathbf{v}_{E}-\nabla \cdot\left(\rho \mathbf{v}_{\perp}^{(1)}\right)$, which by means of Eqs. (12) and (15) reduces to

$D_{t} \rho=\frac{2}{R B}\left(\rho \frac{\partial \phi}{\partial z}-\frac{m_{i}}{e} \frac{\partial p}{\partial z}\right)-\frac{m_{i} T_{e}}{\eta e^{2}} \nabla_{\|}^{2}\left(\frac{e \phi}{T_{e}}-\ln \rho\right)+R_{n}$,

where $R_{n}=\left(v_{e n} / \omega_{c e} B\right)\left[\left(m_{i} / e\right) \nabla_{\perp}^{2} p-\nabla_{\perp} \cdot\left(\rho \nabla_{\perp} \phi\right)\right]$. In the limit of $\epsilon_{A} \rightarrow 0$ the first terms on the right in Eqs. (15) and (16) vanish, hence to first order in the small parameter $\epsilon_{A}$ these terms represent the effect of toroidicity.

If drift-waves are not excited, the plasma perturbations are "flute-like" and an isothermal assumption is generally not justified. In this case Eqs. (15) and (16) should be complemented with an energy equation, describing the evolution of $T_{e}$. Often it is appropriate to neglect heat conduction in this energy equation, leaving us with the adiabatic equation of state, Eq. (9), which combined with the mass conservation equation (8) takes the form $\left(\partial_{t}+\mathbf{v} \cdot \nabla\right) p=$ $-\gamma p \nabla \cdot \mathbf{v}$. By means of Eq. (10) this can be written as
$D_{t} p=-\gamma p \nabla \cdot \mathbf{v}_{E}-\gamma p \nabla \cdot \mathbf{v}_{\perp}^{(1)}-\mathbf{v}_{\perp}^{(1)} \cdot \nabla p$, which reduces to

$D_{t} p=\frac{2 \gamma p}{R B} \frac{\partial \phi}{\partial z}+\frac{\gamma p}{B \omega_{c i}} D_{t} \nabla_{\perp}^{2} \phi$

$+\frac{1}{B \omega_{c i}} \nabla_{\perp} p \cdot D_{t} \nabla_{\perp} \phi+Q_{n}$,

where $Q_{n}=\left(v_{i n} / \omega_{c i} B\right)\left(\nabla_{\perp} p \cdot \nabla_{\perp} \phi+\gamma p \nabla_{\perp}^{2} \phi\right)$. The drift approximation has allowed us to reduce the numbers of equations and variables from nine in the full electrostatic model to the three Eqs. (15)-(17) in the three scalar variables $\phi, p$, and $\rho$. This represents a considerable simplification, which is important for the feasibility of numerical simulations as well as for interpretation of simulations and experiments.

For numerical computation Eqs. (15)-(17) have been formulated in the cylindrical coordinates. Only axisymmetric perturbations (flute modes) are considered $(\partial / \partial \varphi=0)$ so the problem reduces to $2 \mathrm{D}$ on a poloidal cross section of the plasma. The resulting equations are solved numerically by means of a finite difference scheme on a square $0.3 \mathrm{~m}$ $\times 0.3 \mathrm{~m}$, and the major radius is $R_{0}=0.6 \mathrm{~m}\left(\epsilon_{A}=0.25\right)$. A source term in charge, mass and pressure simulates the effect of emissive cathode located at the center of the cross section. The spatial distribution of the source intensity is a 2-D circularly symmetric Gaussian with standard deviation $0.03 \mathrm{~m}$. Dirichlet boundary contition have been imposed $\left(\phi=\varrho=0, \rho=\rho_{b}=\right.$ const . , and the effect of a circular poloidal limiter near the wall has been modelled as a sink that removes excessive charge and plasma in the limiter region.

Simulations with the geometry described above will be referred to as global. This model describes the experimental situation quite realistically, but since a finite difference code is employed, the code is slow and spatial resolution and accuracy are rather poor. More accurate and faster codes can be obtained by employing a slab geometry, implementing periodic boundary conditions in the $z$-direction. This scheme still assumes a plasma source along a vertical (along $z$ ) strip in the center of the slab (around $R=R_{0}$ ) and a sink (limiter) at $R=R_{0} \pm a$. This model retains the effect of magnetic field curvature, but charge injection from the source will lead to vertical sheared flow instead of a poloidal rotation. Although these local simulations cannot realistically describe the global features of the laboratory experiment, they are more easily linked to analytic theories for the interchange instabilitiy. Some results from both classes of simulations will be presented in Sect. 5 .

\section{The problem of equilibrium}

In plasma physics the concept of equilibrium has many different meanings, which sometimes leads to some confusion. Full thermodynamic equilibrium is rarely attained because of the long time scales involved in the relaxation of energy between electrons and ions. In magnetically confined plasmas the time-averaged forces on a fluid element balance each 
other, i.e. the plasma exhibits an MHD equilibrium. If the plasma does not attain a stable equilibrium of this type, it is assumed that the fluid is subject to rapid expansion due to the pressure forces and only weak pressure gradients can persist. This picture, however, is too crude to cover all interesting laboratory and astrophysical plasmas. Even in the absence of MHD equilibrium magnetic fields can provide considerable confinement compared to a field free expansion, although the optimal confinement required in magnetic fusion plasmas cannot be obtained. This is the case with the simple torus configuration, where the magnetic field effectively prevents expansion along the minor radius, but expansion along the major radius can only be prevented if wall currents are part of the plasma current system.

\subsection{Analysis of charge balance}

If the wall of the vacuum vessel were electrically insulating, plasma currents in the $z$ direction due to gradient and curvature drift of electrons (assume cold ions) would lead to charge accumulation of opposite sign on the top and bottom vessel wall. Note that this current $\mathbf{j}_{B}=(-2 p / B R) \hat{\mathbf{z}}$ due to guiding center drifts is related to the current density due to fluid drifts through the relation $\mathbf{j}_{p}=\mathbf{j}_{B}+\mathbf{j}_{M}$, where $\mathbf{j}_{M}=\nabla \times\left(p \mathbf{B} / B^{2}\right)$ is the magnetization current. The latter, however, does not contribute to charge accumulation on the wall. Note also that $\mathbf{j}_{B}$ is equivalent to a current due to gravitational drift from a centrifugal acceleration $g_{c}=2 T_{e} / R m_{i}$. The charge accumulation leads to a polarization electric field and to an oppositely directed $j_{m}=\rho d_{t} E / B^{2}$. The surface charge density is given by $d_{t} \sigma=j_{B}+j_{m}$, and since $E=-\sigma / \epsilon_{0}$, we get $d_{t} E=\left(\rho g_{c} / \epsilon_{0} B\right)-\left(\rho / \epsilon_{0}\right) B^{2} d_{t} E$, and hence the electric drift along the major radius becomes

$\mathbf{v}_{R}=\frac{E}{B}=\frac{g_{c}}{1+\chi^{-1}} t \approx g_{c} t$

where $\chi^{-1}=\epsilon_{0} B^{2} / M n$ is much smaller than unity for most laboratory plasmas of interest. Conventional wisdom is that this "free fall" of the plasma in the centrifugal force field makes the simple torus plasma essentially unconfined by the magnetic field. As the reasoning above indicates, however, this is not necessarily true if charge accumulation is prevented by a conducting boundary, i.e. if the vertical plasma currrent is closed by a wall current. Such a wall current implies that there is a Lorentz force on the wall, and hence (by Newton's 3. law) that the vacuum vessel can act on the plasma by a force different from the kinetic pressure due to direct wall contact.

\subsection{Analysis of force balance}

The reasoning above is quite typical for the electrostatic model. The centrifugal force provides a non-solenoidal current density, and the charge continuity equation requires an additional inertia driven current density which implies a growing electric field and a corresponding accelerated drift velocity. It is also possible to show the same by considering directly the force balance, and without assuming low beta and the electrostatic approximation. Consider for simplicity a slab like plasma $\partial_{\phi}=\partial_{z}=0$. Assume that we have walls at $R=R_{1}$ and $R=R_{2}$, and let us separate the currents in the $z$-direction into plasma currents $j_{p l}$ and wall currents $j_{w}$,

$j=j_{p l}+\frac{I_{w}^{(1)}}{2 \pi R_{1}} \delta\left(R-R_{1}\right)+\frac{I_{w}^{(2)}}{2 \pi R_{2}} \delta\left(R-R_{2}\right)$,

where $I_{w}^{(1,2)}$ are the currents in the inner and outer wall, respectively. Assume that the plasma currents and the wall currents constitute a closed circuit (no external circuit), i.e. that $I_{p l}+I_{w}=0$, where $I_{p l}=2 \pi \int j_{p l} R d R$ and $I_{w}=$ $I_{w}^{(1)}+I_{w}^{(2)}$. From the $R$-components of Ampère's law and the momentum equation we get

$\frac{d p}{d R}=j_{p l} B=\left(j_{w}-j\right) B=j_{w} B-\frac{B}{\mu_{0} R} \frac{d}{d R}(R B)$.

Multiplication of this equation by $R^{2}$, and integration over the closed interval $\left[R_{1}, R_{2}\right]$ including the wall current sheets, yields

$2 \int_{R_{1}}^{R_{2}} p R d R=\left[R^{2} p\right]_{R_{1}}^{R_{2}}+\frac{R_{0} B_{0}}{2 \pi} I_{w}$.

Here we have integrated by parts and used that $I_{p l}+I_{w}=$ $0 \Rightarrow B_{1} R_{1}=B_{2} R_{2}=R_{0} B_{0}$, where $B_{0}$ is the magnetic field generated by the external field coils at $R=R_{0}$. Equation (21) shows that the internal plasma pressure can be balanced by kinetic pressure due to wall contact or by forces due to wall currents. The latter is of course preferable if one wants to confine the plasma. Let us consider a pressure profile that peaks near the center $R_{0}=\left(R_{1}+R_{2}\right) / 2$ and is zero at the left and right boundaries. The diamagnetic current density $j_{p}=-B^{-1} d p / d r$ is in the negative $\hat{\mathbf{z}}$-direction to the left of the peak, and in the postive direction to the right. Let $I_{p l}^{(1)}<0$ and $I_{p l}^{(2)}>0$ be the plasma currents to the left and right of the peak, respectively. If $\epsilon_{A}=a / R_{0} \ll 1$, we have that $\left|I_{p l}^{(1)}\right| \approx\left|I_{p l}^{(2)}\right| \sim 4 \pi R_{0}\langle p\rangle / B_{0}$, where $\langle p\rangle$ is the spatially averaged pressure. On the other hand Eq. (21) shows that $\left|I_{w}\right|=4 \pi a\langle p\rangle / B_{0}$, hence $I_{w} \sim \epsilon_{A} I_{p l}^{(1,2)}$. This shows that for a large aspect ratio torus $\left(\epsilon_{A} \ll 1\right)$ the wall current is a small fraction of the current circulating in the plasma.

Even though the global charge/force balance can be attained by wall currents, local balance cannot generally be attained in a stationary plasma without flow. Without collisions and flow the total current density is diamagnetic, $\mathbf{j}_{\perp}=\mathbf{j}_{p}$, and we find that $\nabla \cdot \mathbf{j}_{\perp}=-(2 / B R) \partial_{z} p$. If the pressure profile is peaked at the center of the plasma, and there is no charge injection, there will be accumulation of charge of opposite charge at the lower and upper part of the vacuum vessel, and hence an $\mathbf{E} \times \mathbf{B}$-flow along the major radius close to the equatorial plane. Local balance, and hence a static equilibrium, can only be attainded in the special case of a slab configuration, $\partial_{z} p=0$.

Global simulations of the more general case of a pressure profile peaked at the center also in the $z$-direction show that 
most of the plasma flows back close to the lower and upper wall, setting up a stationary double vortex flow where the plasma produced in the center is transported to the limiter region by advection along the major radius. This shows the existence of stationary flows where the charge accumulation due to $\mathbf{j}_{p}$ is compensated by the inertial current $\mathbf{j}_{m}$ and the Pedersen current $\mathbf{j}_{v}$. The flow equilibria from these simulations agree quite well with our experimental equilibria for microwave (ECR) plasma (Rypdal et al., 1997) or for some hot cathode discharges in heavy ion plasma (Paulsen et al., 2000). For more typical hot cathode discharges one has a deep concentric potential well which implies a sheared poloidal rotation of the plasma. As will be discussed in the next section this rotating state is always fluctuating, because only anomalous mechanisms can provide the necessary radial plasma transport.

\section{Instabilities and critical gradients}

\subsection{Instabilities and thresholds}

A local stability analysis of flute modes and drift waves in a slab with magnetic field of radius of curvature $R_{0}$ and exponential density profile $n \sim \exp \left[\left(R_{0}-R\right) / L_{n}\right]$ has been performed by Garcia (2001). For a plasma without a sheared flow, flute modes $\left(k_{\|}=0\right)$ are unstable only on the outside density slope, where the density gradient points in the opposite direction of the radius of curvature of the magnetic field. The instability condition for a mode with vertical wavenumber $k_{z}$ and $k_{R}=0$ is $\epsilon \equiv R_{0} / 2 L_{n}>1+\rho_{s}^{2} k_{z}^{2} / 4$, where $L_{n}$ is the density gradient scale length and $\rho_{s}=c_{s} / \omega_{c i}$ is the Larmor radius of an ion with electron temperature. Smaller $k_{z}$ are more unstable than larger, but since the smallest possible wavenumber in a slab with vertical extent $L_{z}$ is $k_{z}=2 \pi / L_{z}$, the instability condition becomes $L_{n}<L_{t h r}$, where

$L_{t h r}=\frac{R_{0}}{2\left(1+\pi^{2} \rho_{s}^{2} / L_{z}^{2}\right)} \approx \frac{R_{0}}{2}$.

At the threshold $L_{n}=L_{t h r}$ the smallest possible wavenumber is marginally stable and all higher wavenumbers are linearly stable. Stable and marginally stable flute-modes have $\alpha_{k}^{(n \phi)}=\pi$.

The threshold for the flute interchange instability is due to the stabilizing effect of the compression term $-\rho \nabla \cdot \mathbf{v}_{E}=$ $(2 \rho / R B) \partial_{z} \phi$ on the right hand side of the mass continuity equation (17). This term, and the corresponding threshold, is due to magnetic field curvature and does not occur in the gravitational interchange instability.

While flute modes are driven unstable by field curvature on the weak field side (i.e. on the outside slope) of the torus cross section, drift waves do not require field curvature for instability, and can be unstable both on the outside and inside slopes. However, on the outside slope the compression term creates the same threshold as for flute modes.

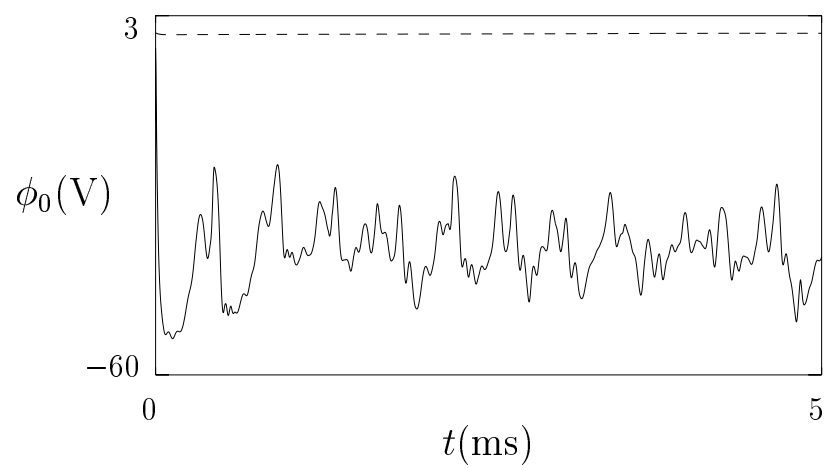

Fig. 1. Time evolution of potential minimum $\phi_{0}$ for Hydrogen discharge (full line) and Argon discharge (broken line).

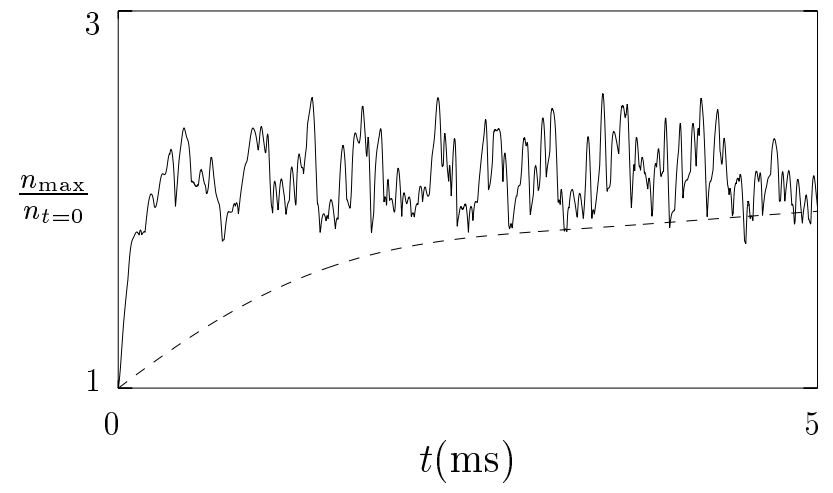

Fig. 2. Time evolution of $n_{\max } / n_{0}$ for Hydrogen discharge (full line) and Argon discharge (broken line).

For drift-waves the growth rate and cross phase $\alpha_{k}^{(n \phi)}$ depend on the parameter

$\psi=k_{\|}^{2} L_{n} \rho_{s} \frac{\omega_{c e}}{v_{e}} \frac{\left(1+k_{z}^{2} \rho_{s}^{2}\right)^{2}}{k_{z}^{3} \rho_{s}^{3}}$,

the maximum growth rate ocurring at $\psi \approx 1$. In the hydrodynamic limit $\psi \ll 1$ one finds that $\alpha_{k}^{(n \phi)} \rightarrow \pi / 4$, $n_{k} / n_{0} \gg e \phi_{k} / T_{e}$, and the growth rate $\gamma_{k} \propto k_{\|}^{2} / v_{e}$. In the adiabatic limit $\psi \gg 1$ we have $\alpha_{k}^{(n \phi)} \rightarrow 0, n_{k} / n_{0} \approx e \phi_{k} / T_{e}$, and $\gamma_{k} \propto v_{e} / k_{\|}^{2}$. The smallest possible parallel wavenumber in the toroidal geometry is $k_{11}=1 / R_{0}$. For the experimental parameters of the Blaamann device the maximum growth rate $(\psi \approx 1)$ occurs for $k_{z} \rho_{z} \approx 1$, i.e. for perpendicular wave-lengths $\lambda_{\perp} \approx 2 \mathrm{~cm}$. Most of the power is in larger wave-lengths $\left(k_{z}^{2} \rho_{s}^{2} \ll 1\right)$, corresponding to the adiabatic regime $\psi \gg 1$.

The results quoted here are valid for a situation without a shear flow. Studies of the gravitational instability indicate that velocity shear should increase the stability threshold, an some results exist also for curvature driven flute eigenmodes in a slab geometry with prescribed velocity profiles (Mahajan et al., 1997). The relevance of these results to the toroidal geometry is not clear, however. 

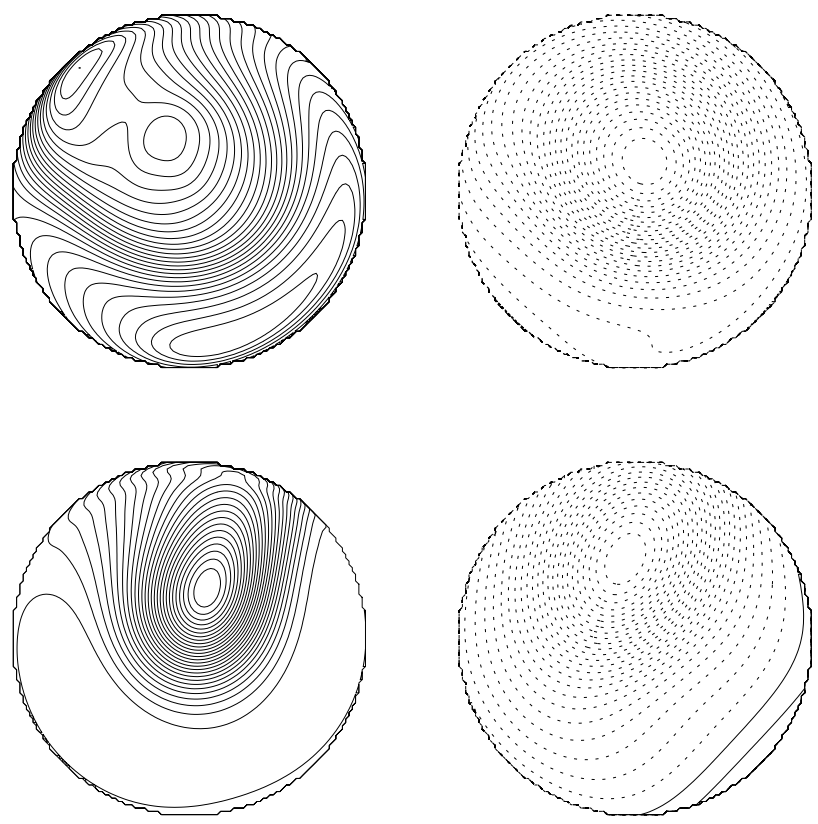

Fig. 3. Time averaged equidensity contours (left) and equipotential contours (right) for global simulations of Hydrogen discharge (top) and Argon discharge (bottom). The equipotential contours represent flow surfaces for the $\mathbf{E} \times \mathbf{B}$-flow and show closed flow surfaces through the source region for the Hydrogen discharge, but open flow surfaces for the Argon discharge, allowing for passive advection of plasma frome source to wall in the latter case.

\subsection{Global simulations and experimental profiles}

Global simulations of poloidally rotating plasmas in the flute limit $\left(k_{\|}=0\right)$ typically show two transient time scales, one short for the formation of the potential structure and one longer for saturation of the density growth. The latter is basically the particle confinement time. During a time of order $v_{i n}^{-1} \sim 0.1 \mathrm{~ms}$ after onset of the sources the growth of the potential well has saturated. This saturation is shown for a Hydrogen discharge by the full curve in Fig. 1, and for an Argon discharge with higher plasma density by the broken curve. The potential well for Hydrogen is about $40 \mathrm{~V}$ deep, giving rise to flow surfaces which are closed around the source. For Argon the well depth is less than $1 \mathrm{~V}$, giving rise to a situation with open averaged flow surfaces traversing the source region, allowing stationary convective transport. Flow surfaces for the two cases are shown in Fig. 3.

Figure 2 shows that the density continues to grow for $0.5 \mathrm{~ms}$ in the Hydrogen case and for around $5 \mathrm{~ms}$ in the Argon case. The slower growth in the Argon simulation is due to the fact that the initial background electron number density $n_{0}$ was set 10 times higher than for Hydrogen, while the ionization source was the same. This means that it takes 10 times longer to build up the same density scale length $L_{n}$ (or equivalently the same ratio, $n_{\max } / n_{0}$, where $n_{\max }$ is the maximal number density over the cross section of the plasma column).

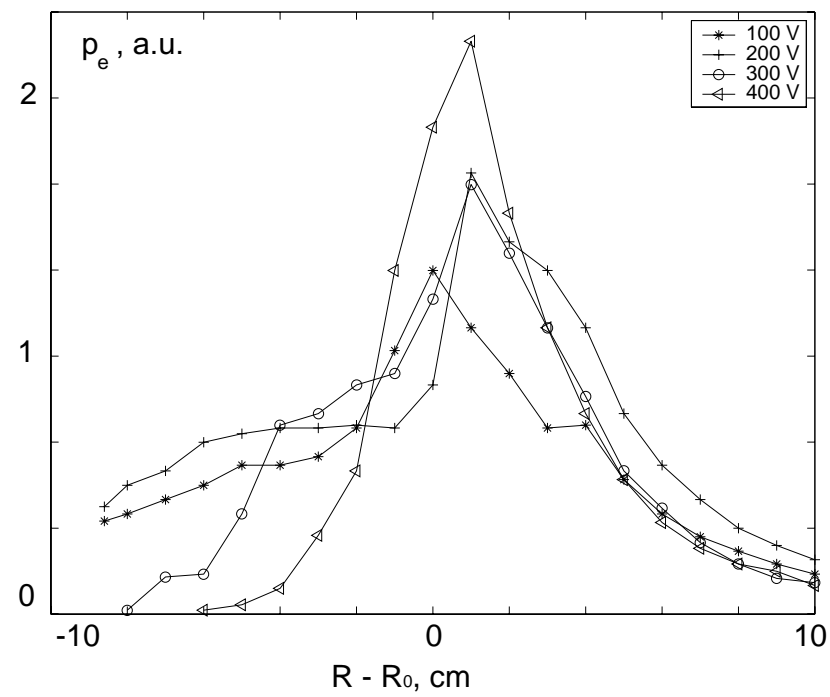

Fig. 4. Measured electron pressure profiles profiles for different values of the discharge voltage, $V_{d i s}=100,200,300,400 \mathrm{~V}$. Ionization source is roughly proportional to $V_{d i s}$.

A technical feature of the simulations shown here is that an initial, spatially homogeneous number density $n_{0}$ has been chosen, and the limiter is modelled in such a way that the density in the limiter region never deviates much from this value. In practice this means that $n=n_{0}$ works as a boundary condition. An interesting empirical observation from many simulations is that the time-average $\left\langle n_{\max }\right\rangle / n_{0}$ seems to saturate at a value around 2.4 for the turbulent states with closed flow surfaces, and around 2.0 for the quiescent states with stationary flow along open surfaces.

It could be tempting to interpret the universality of the scale length $L_{n}$ for turbulent states as the manifestation of a linear stability threshold, and that these states are marginally stable flow equilibria. Marginal stability, however, requires the existence of neighbouring stable states. In the simulation of turbulent states, however, the contribution to $\nabla \cdot \mathbf{j}_{m}$ from the turbulent stress is a significant contribution to the charge balance, and this indicates that the time-averaged concentric flow states are not equilibria, and thus cannot correspond to marginal stability. Thus, it is conceivable that the universal value of $L_{n}$ is a characteristic property of the nonlinear saturated states in this geometric configuration rather than a manifestation of a linear stability threshold.

Experimental electron pressure profiles for different magnitudes of the plasma source (discharge voltage) are shown in Fig. 4. The radial profile on the outside slope seems to be close to exponential, and the scale length is nearly independent of the source strength. The pressure gradient is almost ten times steeper than that corresponding to the linear instability threshold in a plasma without shear flow given by Eq. (22), but agrees well with the gradient scale length observed in the global simulations. 


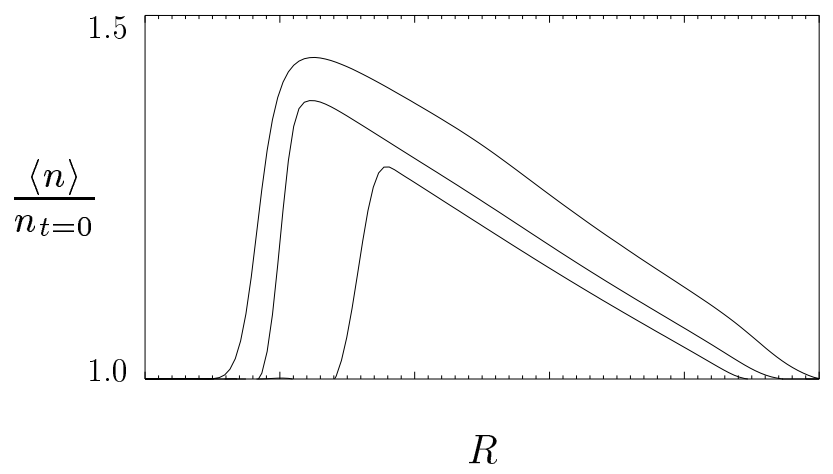

Fig. 5. Electron density profiles from local simulations without charge source for plasma source of strength 1,10 , and 100, respectively.

\subsection{Local simulations}

Criticality of gradients are observed more clearly in local simulations in slab geometry, where sources and limiters are vertical strips (in $z$-direction), and we have periodic boundary conditions in this direction. This provides a faster code, but cannot provide poloidal rotation of the plasma. On the other hand, this geometry provides the existence of an equilibrium, which gives clearer meaning to the concept of stability.

Without charge source the density gradient on the outside slope is very close to (slightly above) the one corresponding to the linear stability threshold, and this feature is very insensitive to the source strength. Radial profiles for different source strengths (but without sheared flows) are shown in Fig. 5. The profiles on the outside slope are nearly exponential and $L_{n}$ is almost independent of the source and very close to the linear threshold $L_{t h r}=R_{0} / 2$.

Spatial spectral analysis of the turbulent particle flux shows that the largest scales (smallest wavenumbers) are responsible for most of the total flux. The cross phase $\alpha_{k}^{(n \phi)}$ is somewhat above $\pi\left(\alpha_{k}^{(n \phi)}=\pi\right.$ yields zero anomalous flux), and power spectra are scale invariant (have power-law dependence $\sim f^{-\alpha}$ ). Slab simulations with charge source yields a potential well and sheared flow in the $z$-direction. Like the non-slab simulations the saturated gradient now becomes much steeper than the linear threshold for unsheared flow. The velocity shear brings the cross-phase $\alpha_{k}^{(n \phi)}$ very close to $\pi$, i.e. very unfavourable for transport, hence requiring stronger fluctuations to sustain a given flux. With a realistic ion-neutral collision frequency introduced in the model, no self-generated zonal flows appear in these simulations. However, if the realistic dissipation terms are replaced with only a diffusion term in the mass continuity equation, zonal sheared flows develop in the absence of a charge source. These flows effectively quench the instability until a stationary, nonturbulent state appears with a purely diffusive density profile.

\section{Experimental results on fluctuations and transport}

A challenging diagnostic problem in the context of crossfield anomalous transport is to perform accurate local measurements of instantaneous particle and energy density fluxes. The basic problem is the following: Suppose the $z-$ axis of a cartesian coordinate system is directed along the ambient magnetic field and we want to measure the anomalous flux in the the $x$-direction. Then simultaneous measurements of instantaneous values of electron density $n$, electric field $E_{y}$ and electron temperature $T_{e}$ are necessary to obtain the anomalous cross-field flux densities. If the dominant modes have cross-phases $\alpha_{n \phi}(\omega)$ close to 0 or $\pi$, the respective fluxes (which are proportional to $\sin \alpha_{n \phi}$ ) become very sensitive to errors in the measurements of $\alpha_{n \phi}$. Such errors will arise for instance if one does not succeed in eliminating the influence of $\tilde{T}_{e}$ on the measurements of $\tilde{V}_{p}$ and $\tilde{n}$. A method to deal with this problem in the context of Langmuir probe measurements was developed by Ratynskaia et al. (2000a,b), and measurements of fluctuations, cross-phases and anomalous fluxes for the simple torus configuration have recently been published by Ratynskaia et al. (2002). The conclusion of these measurements are that flute modes dominate all fluctuations on the outer density slope (on the weak field side), while drift waves might dominate density and potential fluctuations on the inner slope, where flute modes are locally stable. Weak flute modes coexist with the drift waves on the inside slope, manifested through weak temperature fluctuations. The flute mode spectrum exhibits a peak which is due to large scale poloidally rotating structures, probably associated with the lowest poloidal wavenumber $(m=1)$. This is the fastest growing mode according to linear theory for flute interchange instability, but if the gradient is at the threshold for this instability, it also is the only linearly unstable mode. If this is the case, the higher wave-numbers (corresponding to the power-law spectrum at higher frequencies) are nonlinearly driven trough a turbulent cascade. The flux measurements show that the large scale flute mode structures play a substantial role in both particle and energy transport on the outside. On the inside only drift waves contribute to particle transport, while the coupling between temperature fluctuations in flute modes and electric field fluctuations in drift waves yields the major contribution to the energy transport.

\section{Conclusions}

The plasma parameters of many small scale magnetized laboratory devices require mathematical models for description of the low frequency dynamics which are remarkably similar to those employed for description for ionospheric interchange turbulence and field-aligned irregularities. Our electrostatic model derived in Sect. 3.4 (Eqs. 15-17) are generalizations of the model for ionospheric turbulence derived by Huba et al. (1985) and applied to this problem by Hassam et al. (1985). On the other hand this model is also a gener- 
alization of generic models for drift wave turbulence, which has been applied to ionospheric problems as well as laboratory plasmas, including edge turbulence in magnetic confinement devices.

The numerical and experimental studies of plasma potential profiles and potential fluctuations show the existence of both quiet stationary flow states with slow flow along open flow surfaces, and of turbulent flow states with a fast mean poloidal flow. The transition between these types of states has so far not been studied systematically. Time-averaged density (or pressure) profiles indicate the existence of critical profiles in the sense that the gradient scale lengths attain characteristic values that are resilient to variation of the imposed fluxes or other discharge parameters. The absence of a static or stationary flow equilibrium state close to the timeaveraged states observed in global simulations and experiments indicates that this resiliency is not simply a manifestation of a linear stability threshold. Further study is required before the true nature of the critical profiles is properly understood.

Experimental identification of the turbulent modes and measurement of anomalous particle and energy flux densities were briefly reported in Sect. 6 . The modes on the weak field side (outside) were identified as electrostatic flute modes, and hence driven by the interchange instability. On the strong field side these modes are stable, and measurements of crossphases between density and electric field fluctuations, and of dispersion characteristics (phase velocity), indicate that drift waves dominate the density and electric field fluctuations in this region. The possible role of drift waves indicates that parallel electron dynamics should be included in the numerical models, and that 3-D simulation may reveal new physics.

Flux measurements show that both the large scale coherent structures and the power-law part of the turbulent wavenumber spectrum contribute to the total anomalous fluxes. Flute modes and drift waves may both play a role in the transport.

Acknowledgements. This work was supported by the Research Council of Norway under grants 125713/410 and 135373/432.

\section{References}

Block L. P.: Scaling considerations for magnetospheric model experiments, Planet. Space Sci. 15, 1479-1487, 1967.

Demidov, V. I., Ratynskaia, S. V., Armstrong, R. J., and Rypdal, K.: Probe measurements of electron energy distributions in a strongly magnetized low-pressure helium plasma, Phys. Plasmas, 6, 350-358, 1999.
Garcia, O. E.: Two-field transport models for magnetized plasmas, J. Plasma Phys., 65, 81-96, 2001.

Gekelman, W.: Review of laboratory experiments on Alfvén waves and their relationship to space observations, J. Geophys. Res., 104, 14 417-14 435, 1999.

Goldston, R. J. and Rutherford, P. H.: Introduction to Plasma physics, IoP publishing, 1995.

Hysell, D. L.: An overview and synthesis of plasma irregularities in equatorial spread F, J. Atmos. Terr. Phys., 62, 1037-1056, 2000.

Hassam, A. B., Hall, W., Huba, J. D., and Keskinen, M. J.: Spectral characteristic of interchange turbulence and chaotic behaviour, J. Geophys. Res. 91, 13 513-13 522, 1986.

Huba, J. D., Hassam, A. B., Schwartz, I. B., and Keskinen, M. J.: Ionospheric turbulence: Interchange instabilities and chaotic behaviour, Geophys. Res. Lett., 12, 65-68, 1985.

Mahajan S., Singh, R., and Avinash, K.: Theory of plasma confinement in devices with pure toroidal field, Phys. Plasmas, 4, 2612-2618, 1997.

Motley, R. W.: Q Machines, Academic Press, 1975.

Paulsen J.-V., Rypdal, K., Garcia, O. E., and Olsen, O. M.: Anomalous transport in the simple torus, Physica Scripta, T84, 203-205, 2000.

Ratynskaia, S. V., Demidov, V. I., and Rypdal, K.: A probe for measurement of electrostatic fluctuations in a low-temperature magnetized plasma, Rev. Sci. Instr, 71, 1367-1369, 2000.

Ratynskaia, S. V., Demidov, V. I., and Rypdal, K.: Probe measurements of electron temperature and density in strongly magnetized plasma, Rev. Sci. Instr, 71, 3382-3384, 2000.

Ratynskaia, S. V., Demidov, V. I., and Rypdal, K.: Measurements of anomalous particle and energy fluxes in a magnetized plasma, Phys. Rev. E. 65, 066403-1-066403-9, 2002.

Rypdal, K. and Brundtland, T.: The Birkeland terella experiments and their importance for the modern synergy of laboratory and space plasma physics, J. Phys., 7, C4-113-131, 1997.

Rypdal, K., Garcia, O. E., and Paulsen, J.-V.: Anomalous CrossField Current and Fluctuating Equilibrium of Magnetized Plasmas, Phys. Rev. Lett., 79, 1857-1860, 1997.

Rypdal, K., Grønvoll,E., Øynes, F., Fredriksen, Å, Armstrong, R. J., Trulsen, J., and Pécseli, H. L., Confinement and turbulent transport in a simple magnetized torus, Plasma Phys. Contr. Fusion, 36, 1099-1114, 1994.

Rypdal, K., Fredriksen, H., Paulsen, J.-V., and Olsen, O. M.: Coherent structures in the turbulent equilibrium of the simple magnetized torus, Physica Scripta, T63, 167-173, 1996.

Rypdal, K., Fredriksen,Å, Olsen, O. M., and Hellblom, G.: Microwave-plasma in a simple magnetized torus, Phys. Plasmas, 4, 1468-1480, 1997.

Øynes, F., Olsen, O. M., Pécseli, H. L., Fredriksen, Å., and Rypdal, K.: Experimental study of low-frequency electrostatic fluctuations in a magnetized toroidal plasma, Phys. Rev. , E57, 22422255, 1998.

Øynes, F., Pécseli, H. L., and Rypdal, K.: Fluctuations in a magnetized toroidal plasma without rotational transform, Phys. Rev. Lett., 75, 81-84, 1995. 